\title{
NHS Breast Screening multidisciplinary working group guidelines for the diagnosis and management of breast lesions of uncertain malignant potential on core biopsy (B3 lesions)
}

DOI:

10.1016/j.crad.2018.04.004

Document Version

Accepted author manuscript

Link to publication record in Manchester Research Explorer

Citation for published version (APA):

Pinder, S. E., Shaaban, A., Deb, R., Desai, A., Gandhi, A., Lee, A. H. S., Pain, S., Wilkinson, L., \& Sharma, N. (2018). NHS Breast Screening multidisciplinary working group guidelines for the diagnosis and management of breast lesions of uncertain malignant potential on core biopsy (B3 lesions). Clinical radiology, 73(8), 682-692. https://doi.org/10.1016/j.crad.2018.04.004

Published in:

Clinical radiology

\section{Citing this paper}

Please note that where the full-text provided on Manchester Research Explorer is the Author Accepted Manuscript or Proof version this may differ from the final Published version. If citing, it is advised that you check and use the publisher's definitive version.

\section{General rights}

Copyright and moral rights for the publications made accessible in the Research Explorer are retained by the authors and/or other copyright owners and it is a condition of accessing publications that users recognise and abide by the legal requirements associated with these rights.

\section{Takedown policy}

If you believe that this document breaches copyright please refer to the University of Manchester's Takedown Procedures [http://man.ac.uk/04Y6Bo] or contact uml.scholarlycommunications@manchester.ac.uk providing relevant details, so we can investigate your claim.

\section{OPEN ACCESS}




\section{Clinical Radiology}

\section{Guidelines for the diagnosis and management of breast lesions of uncertain malignant potential on core biopsy (B3 lesions): The NHS Breast Screening multidisciplinary working group \\ --Manuscript Draft--}

\begin{tabular}{|c|c|}
\hline Manuscript Number: & CRAD-D-18-00279R1 \\
\hline Full Title: & $\begin{array}{l}\text { Guidelines for the diagnosis and management of breast lesions of uncertain malignant } \\
\text { potential on core biopsy (B3 lesions): The NHS Breast Screening multidisciplinary } \\
\text { working group }\end{array}$ \\
\hline Article Type: & Original Paper \\
\hline Corresponding Author: & $\begin{array}{l}\text { Nisha Sharma, MBChB } \\
\text { Leeds teaching hospital NHS Trust } \\
\text { Leeds, UNITED KINGDOM }\end{array}$ \\
\hline \multicolumn{2}{|l|}{$\begin{array}{l}\text { Corresponding Author Secondary } \\
\text { Information: }\end{array}$} \\
\hline Corresponding Author's Institution: & Leeds teaching hospital NHS Trust \\
\hline \multicolumn{2}{|l|}{$\begin{array}{l}\text { Corresponding Author's Secondary } \\
\text { Institution: }\end{array}$} \\
\hline First Author: & Nisha Sharma, MBChB \\
\hline \multicolumn{2}{|l|}{ First Author Secondary Information: } \\
\hline \multirow[t]{9}{*}{ Order of Authors: } & Nisha Sharma, MBChB \\
\hline & Sarah Pinder \\
\hline & Abeer Shaaban \\
\hline & Rahul Deb \\
\hline & Anil Desai \\
\hline & Ashu Gandhi \\
\hline & Andrew H S Lee \\
\hline & Simon Pain \\
\hline & Louise Wilkinson \\
\hline \multicolumn{2}{|c|}{ Order of Authors Secondary Information: } \\
\hline Abstract: & $\begin{array}{l}\text { Needle core biopsy is considered the histological diagnostic method of choice for } \\
\text { screen detected breast lesions. Although the majority are definitively diagnosed as } \\
\text { normal, benign or malignant, approximately } 7 \% \text { are categorised as B3, of uncertain } \\
\text { malignant potential. These include a wide range of lesions with different risks of } \\
\text { associated malignancy from <2\% to approaching } 40 \% \text { from literature review in UK } \\
\text { practice. Historically these have typically been surgically excised as a diagnostic } \\
\text { procedure but the majority are then proven to be benign. An alternative approach, for } \\
\text { many of these lesions, is thorough sampling/excision by vacuum-assisted biopsy } \\
\text { techniques to exclude the presence of co-existing carcinoma. This would potentially } \\
\text { reduce the benign open biopsy rate whilst maintaining accuracy of cancer diagnosis. A } \\
\text { group from the Radiology, Surgery and Pathology NHS Breast Screening Programme } \\
\text { Co-ordinating Committees and an additional co-opted expert were charged with review } \\
\text { and development of guidelines for the clinical management of the B3 lesions. The } \\
\text { guidelines reflect suggested practice as stated by the NHS Breast Screening } \\
\text { Programme and approved by the RCR. }\end{array}$ \\
\hline
\end{tabular}


Guidelines for the diagnosis and management of breast lesions of uncertain malignant potential on core biopsy (B3 lesions): The NHS Breast Screening multidisciplinary working group

Authors: Sarah E Pinder ${ }^{1}$, Abeer Shaaban ${ }^{2}$, Rahul Deb ${ }^{3}$, Anil Desai ${ }^{3}$, Ashu Gandhi ${ }^{4}$, Andrew HS Lee ${ }^{5}$, Simon Pain ${ }^{6}$, Louise Wilkinson ${ }^{8}$, Nisha Sharma ${ }^{9}$.

1. Prof Sarah E Pinder, Professor of Breast Pathology, King's College London, Guy's Hospital, London. SE1 9RT.

2. Dr Abeer Shaaban, Consultant Histopathologist, University Hospitals Birmingham NHS Foundation Trust, Mindelsohn Way, Birmingham. B15 2WB.

3. Dr Rahul Deb, Consultant Histopathologist, Royal Derby Hospital; Uttoxeter New Road, Derby. DE22 3NE.

3. Mr Anil Desai, Consultant Oncoplastic and Reconstructive Breast Surgeon, Breast Care, Chartwell Unit, Princess Royal University Hospital, Farnborough Common, Kent. BR6 8ND.

4. Mr Ashu Gandhi, Consultant Breast \& Endocrine Surgeon, University Hospital of South Manchester, Manchester. M23 9LT.

5. Dr Andrew HS Lee, Consultant Histopathologist, Nottingham University Hospitals NHS Trust, Nottingham City Hospital, Nottingham. NG5 1PB.

6. Mr Simon Pain, Consultant Surgeon, Norfolk and Norwich University Hospital; Colney Lane, Norwich. NR4 7UY. 
8. Dr Louise Wilkinson, Consultant Radiologist, St George's Hospital, Blackshaw Road, Tooting, London. SW17 OBZ.

9. Dr Nisha Sharma, Consultant Radiologist, Breast Unit, Level 1 Chancellor Wing, St James Hospital, Leeds. LS9 7TF.

Address for correspondence: Dr Nisha Sharma, Leeds/Wakefield Breast Screening unit, Seacroft Hospital, York Road, Leeds, LS79TF. Email: nisha.sharma2@nhs.net

Running Title: Management of B3 lesions. 


\section{Author Contributions}

Authors are required to identify the contributions for which they are responsible. The author responsible for the integrity of the entire study should be identified. Please list the following phrases and beside each indicate the name(s) of the author(s) to whom they apply:

1 guarantor of integrity of the entire study N. Sharma, S. Pinder

2 study concepts and design N.Sharma, S.Pinder, A.Shaaban,

L.Wilkinson, R.Deb, A.Gandhi, A.Lee, S.Pain, A.Desai

3 literature research S.Pinder

4 clinical studies N/A

5 experimental studies / data analysis N/A

6 statistical analysis N/A

7 manuscript preparation S.Pinder and N.Sharma

8 manuscript editing N.Sharma

Items that do not apply should also be indicated with N/A. Where there is any uncertainty regarding authorship the editor of the study reserves the right to contact the guarantor of the study for further information. 
a few minor points:

I do not want to change text etc. as these guidelines have been approved by the working group and RCR so should be left largely unchanged.

Highlights are not required for Guidelines in my view and so can be ignored. Response: Thank you

The figures 1-6 - flow diagrams - are difficult to read and there appear to be small boxes scattered all over - can this be tidied up. Response: I have uploaded the figures so that may help and also the text is unchanged so just need to replace the figures in the document with those uploaded

The references are simply listed - is it possible to number them and add them to the text at appropriate points rather than having the author name etc. in keeping with the journal format. Response: Apologies - all sorted

I think it would be a good idea at the end of the title to insert a colon: and add the name of the working group Breast Screening whatever and maybe somewhere in the summary mention these guidelines reflect suggested practice as stated by the Breast group and approved by the RCR. Response: Added it to the title and have stated in the abstract and summary about NHS breast screening and approved by RCR. 
Breast Special Issue (part 2)

NHS Breast Screening Multidisciplinary Working Group Guidelines for the diagnosis and management of breast lesions of uncertain malignant potential on core biopsy (B3 lesions)

S. E. Pinder ${ }^{1}$, A. Shaaban² , R. Deb³, A. Desai ${ }^{4}$, A. Gandhi ${ }^{5}$, A. H. S. Lee ${ }^{6}$, S. Pain 7 , L. Wilkinson ${ }^{8}$, N. Sharma ${ }^{9, *}$

1'Department of Breast Pathology, King's College London, Guy's Hospital, London SE1 9RT, UK

2Department of Histopathology, University Hospitals Birmingham NHS Foundation Trust, Mindelsohn Way, Birmingham B15 2WB, UK

${ }^{3}$ Department of Histopathology, Royal Derby Hospital; Uttoxeter New Road, Derby DE22 3NE, UK

${ }^{4}$ Department of Oncoplastic and Reconstructive Breast Surgery, Breast Care, Chartwell Unit, Princess Royal University Hospital, Farnborough Common, Kent BR6 8ND, UK

${ }^{5}$ Department of Breast \& Endocrine Surgery, University Hospital of South Manchester, Manchester M23 9LT, UK

${ }^{6}$ Department of Histopathology, Nottingham University Hospitals NHS Trust, Nottingham City Hospital, Nottingham. NG5 1PB.

${ }^{7}$ Department of Surgery, Norfolk and Norwich University Hospital, Colney Lane, Norwich NR4 7UY, UK 
${ }^{8}$ Department of Radiology, St George's Hospital, Blackshaw Road, Tooting, London SW17 0BZ, UK

9 Department of Radiology, Breast Unit, Level 1 Chancellor Wing, St James Hospital, Leeds LS9 7TF, UK

*Guarantor and correspondent: N. Sharma, Leeds/Wakefield Breast Screening unit, Seacroft Hospital, York Road, Leeds LS7 9TF, UK.

E-mail address: nisha.sharma2@nhs.net

Running Title: Management of B3 lesions.

\section{ABSTRACT}

Needle core biopsy is considered the histological diagnostic method of choice for screen-detected breast lesions. Although the majority are definitively diagnosed as normal, benign, or malignant, approximately $7 \%$ are categorised as $\mathrm{B} 3$, of uncertain malignant potential. These include a wide range of lesions with different risks of associated malignancy from $<2 \%$ to approaching $40 \%$ from literature review in UK practice. Historically, these have typically been surgically excised as a diagnostic procedure but the majority are then proven to be benign. An alternative approach, for many of these lesions, is thorough sampling/excision by vacuum-assisted biopsy techniques to exclude the presence of co-existing carcinoma. This would potentially reduce the benign open biopsy rate whilst maintaining accuracy of cancer diagnosis. A group from the Radiology, Surgery, and Pathology NHS Breast Screening Programme Co-ordinating Committees and an additional co-opted expert were charged with review and development of guidelines for the clinical management of B3 
lesions. The guidelines reflect suggested practice as stated by the NHS Breast Screening Programme and approved by the Royal College of Radiologists.

\section{INTRODUCTION}

Core biopsy diagnoses from breast abnormalities are classified histologically as B1 (normal) through to B5 (malignant). Although the majority can be definitively diagnosed as normal, benign, or malignant, a proportion, particularly from breast screening, (median 6.7\%, range 3.3-12.6\%) are categorised as B3, lesion of uncertain malignant potential (UK National Health Service Breast Screening Programme [NHS BSP] Pathology Audit, unpublished). Some series have identified a wider range in the proportion of B3 diagnoses amongst different centres, for example Bianchi et al., ${ }^{1}$ reported a range of $4-22 \%$. In the UK, a range of $2.3-7.9 \%$ has been recorded between eight centres ${ }^{2}$ with a consequent range of positive predictive values between 14.3 and $28.3 \%$, indicating a degree of diagnostic variation between pathologists or institutions; however, the B3 category includes a range of lesions, with potential for differences in frequency and patterns of radiological or clinical sampling practice and thus the nature of the abnormalities provided to pathologists. Nevertheless, the lesions are classified as B3 either because: (i) they are entities known to be heterogeneous (and the area sampled by needle core, whilst benign per se, may not be representative of the whole lesion), or (ii) because they are lesions known to be associated with ductal carcinoma in situ (DCIS) or invasive carcinoma, or because both (i) and (ii) apply. A number of other uncommon, miscellaneous lesions are also categorised as B3.

The group of B3 lesions thus include some lesions with epithelial atypia and some without. These are associated with differing risks of "upgrade", defined here as DCIS 
or invasive carcinoma at the same time and at the same site in the breast as the B3 diagnosis. This is not equivalent to "risk" in the sense more commonly used, i.e., risk of development of subsequent invasive carcinoma in any site in either breast; although some lesions that are regarded as B3, of uncertain malignant potential, such as lobular carcinoma in situ (LCIS) or atypical ductal hyperplasia (ADH) confer an increased risk of subsequent carcinoma bilaterally, as well as having a risk of upgrade. The lesions classified as B3 include: atypical intraductal epithelial proliferation (AIDEP); non-pleomorphic/classical lobular (in situ) neoplasia (LN); flat epithelial atypia (FEA); radial scar, with or without epithelial atypia; papillary lesion, with or without epithelial atypia; cellular fibroepithelial lesion where phylloides tumour cannot be excluded; mucocoele-like lesion; and other rare abnormalities, for example, some spindle cell lesions.

Not only are the B3 lesions more often identified through mammographic breast screening than presenting symptomatically, but the overall frequency of diagnosis of the range of $\mathrm{B} 3$ lesions on core biopsy has increased since the advent of the NHS BSP. This may, in part, be because of increased sensitivity of radiographic techniques, for example, the use of digital mammography with its superior detection of low suspicion microcalcification, and because of increased use of core biopsy, including vacuum-assisted biopsy (VAB) samples; however, in addition, some lesions such as FEA were previously not recognised as histopathological entities.

With increasing concern about over-diagnosis and over-treatment through breast screening, which has even led to novel randomised clinical trials of active surveillance of lesions regarded as established malignancy (e.g., LORIS trial; ISRCTN27544579), as well as the more frequent detection of B3 lesions, and the increasing availability of 
wide-bore vacuum-assisted needle biopsy techniques, it was regarded as timely to reconsider the approach to their management. A multidisciplinary group from the NHS BSP was therefore mandated with examination of available data from the literature with the aim of producing guidelines. The group included members of the Radiology (L.W., N.S.), Surgery (A.D., A.G., S.P.), and Pathology (S.E.P., R.D., A.H.S.L.) NHS BSP Co-ordinating Committees, and a co-opted expert (A.S.).

The purpose of this document is not to duplicate available guidance for non-operative pathology specimen handling or reporting, or to act as a diagnostic text for identification of these lesions, although the histological and radiological features of B3 lesions are described briefly for clarity. The group reviewed the literature on upgrade rates for the different types of B3 lesions, with particular concentration on systemic reviews and meta-analyses, along with local and published protocols for their management if available. When available, additional features were considered, including the underlying radiological appearances, the size of cores (14 or $16 \mathrm{G}$ versus wider-bore vacuum-assisted samples such as 7,8 , or $11 \mathrm{G}$ ) and the extent of sampling/numbers of cores, although these were frequently not apparent. The review also included consideration of whether the upgrade rates were influenced by presentation (screen-detected versus symptomatic versus incidental). Although there are limited data on route of presentation of B3 lesions and outcome, there was little evidence that B3 lesions diagnosed through breast screening have a differing upgrade rate to those seen symptomatically or, surprisingly, that the range of lesions is different. ${ }^{3}$

Many series in the literature do not present all of these data and include a range of size of lesions and, significantly, variation in the nature of sampling (e.g., 14 versus $11 \mathrm{G}$ cores); most studies include a mixture of biopsy techniques. In particular, there 
is wide variation in the literature in the proportion of patients who have undergone diagnostic surgical excision for a B3 lesion; it seems undoubtedly the case that those B3 lesions that were excised differed from those that were not surgically removed, lending bias to the data.

\section{DIAGNOSIS AND UPGRADE RATE OF B3 LESIONS}

A variety of lesions are categorised as B3, of uncertain malignant potential. As these have different features histologically and radiologically, as well as varying upgrade rates, each was reviewed separately.

\section{AIDEP}

The term AIDEP is preferred over ADH in core-biopsy samples. ${ }^{4}$ The latter refers to a specific small lesion (microfocal, $<2 \mathrm{~mm}$ or less than two complete spaces in extent) composed of a uniform, small cell, atypical epithelial proliferation either admixed with a non-uniform process, such as usual epithelial hyperplasia, and/or is too small for diagnosis of low-grade DCIS. ADH cannot be definitively diagnosed on the limited sampling provided by core biopsy, as the extent of the lesion cannot be determined with accuracy. AIDEP includes lesions that in a surgical excision would be regarded as $\mathrm{ADH}$ as well as other forms of atypical epithelial proliferation within duct spaces. Apocrine atypia is also classified as AIDEP. There is, therefore, a range of severity of cytological and architectural atypia in the category of AIDEP, from those that are suspicious but insufficient for a definite diagnosis of DCIS, to those that only show a minor degree of atypia.

Clustered microcalcification is the most common radiological abnormality associated with a B3 diagnosis of AIDEP (75\%; 137 of 182 cases $^{5}$ ), with masses and distortions 
equally comprising the remaining lesions. In other series ${ }^{1}$ the proportion of AIDEP presenting with microcalcification is even higher (86\%).

The published literature consistently demonstrates that the upgrade rate of AIDEP to malignancy is greater with small samples (e.g., $14 \mathrm{G}$ cores) compared to VAB specimens. The upgrade rate for AIDEP varies from $18-87 \%$ for $14 \mathrm{G}$ needles compared to $10-39 \%$ with 11 or 9 G samples with a pooled positive predictive value of $21 \%$ from vacuum-assisted sampling. ${ }^{6}$ In essence, unsurprisingly, if a greater amount of tissue is provided, there is a lower chance of "missing" a diagnosis of DCIS or invasive cancer. This reflects the more extensive sampling that is achieved with $V A B$ in this group of lesions in which there is a moderate chance of co-existing malignancy. There are limited data on the upgrade rate for apocrine atypia such that definitive comment cannot be made.

LN

As with AIDEP, the pathologist cannot accurately assess the extent of an atypical intralobular epithelial proliferation in core-biopsy samples. For this reason, the term $\mathrm{LN}$ is preferred in core-biopsy specimens rather than attempting to distinguish atypical lobular hyperplasia (ALH) from LCIS, although these are biologically and genetically equivalent. Classical LN is seen as a low- to intermediate-grade, uniform, intralobular epithelial proliferation of discohesive cells, often with prominent intracytoplasmic lumina. Immunohistochemistry may be utilised on such samples with reduced or negative E-cadherin usually seen, although approximately $10 \%$ show some positivity. ${ }^{7}$ Although classical $\mathrm{LN}$ is categorised as B3 in core biopsy, pleomorphic LCIS is formed from cells with large, more variable nuclei, and often abundant cytoplasm but with similar discohesion and growth pattern as is classified 
as B5a, malignant in situ. Although the clinical behaviour of pleomorphic LCIS is poorly understood at present, it is regarded as comparable to DCIS for the purposes of clinical management, albeit that this is largely based on its biological features rather than long-term follow-up data. LN that is not pleomorphic, but which has comedo-type necrosis or is mass-forming (and which some regard as a variant of pleomorphic LCIS), is most appropriately categorised as B4, suspicious.

LN is usually mammographically occult, but is increasingly found co-incidentally in biopsies of screen-detected lesions, for example in adjacent columnar cell change. Microcalcifications are occasionally seen within classical LN, but are common in the pleomorphic (B5a) form. ${ }^{8}$

Although LN confers an increased risk of development of subsequent invasive carcinoma in either breast, there is also evidence that there is an upgrade of these lesions with co-existing or adjacent DCIS and/or invasive carcinoma. This is the case both for lesions where the $\mathrm{LN}$ appears co-incidental and those where there is, for example, microcalcification in the disease itself. Menon et al., ${ }^{9}$ reported that whilst immediate excision in 25 of 49 patients with LN in core showed malignancy in nine (36\%), a further four of 19 with follow-up of at least 2 years developed malignancy at the site of the core biopsy (21\%), highlighting that $L N$, which is seemingly coincidental to the radiological features, cannot be dismissed.

There is significant variation in the proportion of lesions that have been surgically excised in series in the literature and there is also variation in terminology used (some have attempted to distinguish ALH from LCIS, some have combined the entities as per UK practice ${ }^{4}$ and some series include pleomorphic LCIS). There, therefore, remains significant uncertainty regarding the true upgrade rate associated with $L N$ 
and further data are required;10 however, the upgrade rate overall (with the caveats as above) is $27 \%$ (range $0-60 \%$ ) from systematic literature review. ${ }^{11}$ Given the wide range of upgrade for $\mathrm{LN}$, some groups have attempted to distinguish ALH from LCIS but the range for the former remains wide (0-43\%) compared to the latter $(0-60 \%)$, and therefore, attempting to sub-classify classical LN based on degree of disease in core biopsy appears to be fruitless. ${ }^{11}$ The upgrade of pleomorphic LCIS is, however, higher with $41 \%$ of lesions overall (range $30-60 \%$ ) proving to be adjacent to invasive carcinoma, albeit with smaller numbers in series of this rare entity, supporting its classification as B5a. ${ }^{11}$ Cases of LN with pathological-radiological discordance, pleomorphic cytology or with necrosis, and those with associated AIDEP, have much higher upgrade rates than those with no necrosis and those with radiologicalpathological concordance. ${ }^{12,13}$

\section{FEA}

The terminology for columnar cell lesion has changed over time, causing confusion and difficulties in review of the literature. Originally coming to widespread recognition as columnar alteration with prominent snouts and secretions (CAPPS),${ }^{14}$ the benign forms of columnar cell lesions are now categorised as columnar cell change and/or columnar cell hyperplasia, and these should be reported as B2, benign, and do not require further assessment. ${ }^{4}$ The atypical forms have also been recorded under a range of names including columnar cell atypia, columnar cell hyperplasia with cytological atypia, clinging carcinoma (monomorphic type), atypical cystic duct, ductal intraepithelial neoplasia $1 \mathrm{~b}$ (flat monomorphic type), hypersecretory hyperplasia with atypia, small ectatic ducts lined by atypical ductal cells with apocrine snouts, monomorphic epithelial proliferation, hypersecretory hyperplasia with atypia and 
atypical columnar cell metaplasia. The accepted term for columnar cell lesions with cytological atypia, which is not of high cytonuclear grade, is FEA, and this should be reported as B3. Rarely high cytonuclear grade is present and the process should then be regarded as flat high grade DCIS (B5a). Columnar cell lesions arise in the terminal duct lobular unit, not the larger ducts, and FEA is no exception. The acini are typically mildly to moderately dilated with smooth, rounded internal aspects and usually bear secretions in the luminal space that may bear microcalcification. In the classical form, the acini are lined by small regular, uniform epithelial cells in one or more layers. The nuclei are typically uniform and round, sometimes with speckled chromatin and small nucleoli but without conspicuous mitoses. If there is architectural atypia, in the form of micropapillary structures or bridges, the lesion should be considered within the spectrum of AIDEP in core biopsy, or ADH/low-grade DCIS in excision specimens, rather than FEA.

The columnar cell lesions typically present with microcalcifications, often amorphous, which are seen within secretions in the luminal spaces. The calcifications are identified for biopsy because of their focal and clustered nature, but have no particular distinguishing features to indicate an association with columnar cell change.

The upgrade rate for FEA remains somewhat unclear, as this entity has not been recognised and reported for many decades and has during this time undergone several changes in nomenclature. As with many of the entities included in the B3 category, series are biased with some, but not all, lesions being excised, and others followed-up mammographically and with small numbers in most series. Finally, FEA not infrequently co-exists with AIDEP and the upgrade rate in this setting is higher than FEA alone. ${ }^{15}$ Although initial reports indicated a high risk of associated malignancy, later series note that this is not as prevalent as some of the earlier 
reports suggest. This probably reflects the change in approach by radiologists (sampling smaller lesions with larger needles and better image guidance). Overall, Verschuur-Maes et al., ${ }^{15}$ in a systematic review including 390 of 668 (58\%) where patients had a diagnosis of columnar cell atypia (i.e., FEA) and then surgical excision (within 4 months of the core biopsy specimen) reported that 57 (17\%) had associated carcinoma in the subsequent excision (37 DCIS, 10\%; 20 invasive carcinoma, 4\%). This is essentially similar to UK data from the West Midlands and South Central regions within the NHS BSP where a positive predictive value of $20.8 \%$ for FEA was reported $^{5}$ and with a series from Italy where the upgrade was $12.7 \%$ following $V A B$ sampling. ${ }^{1}$

Radial scar/complex sclerosing lesion (CSL)

These terms are used for lesions of essentially similar appearance but differing in size, radial scar being $<10 \mathrm{~mm}$ in size and CSL being larger lesions. Histologically there is central fibroelastosis containing entrapped benign compressed tubules, typically with more dilated microcystic and fibrocystic changes peripherally. These may be associated with epithelial proliferation, most commonly usual type hyperplasia, but atypical hyperplasia and in situ carcinoma, as well as invasive carcinoma, may be present.

Although small radial scars may be incidental findings, many are detected mammographically as stellate or spiculate lesion with a radiolucent centre and radiating spicules or an architectural distortion. These may be associated with focal microcalcification. Such features cannot reliably distinguish radial scars from low grade cancers; however, if radiology does not show distortion/soft-tissue change, 
then it is probably a coincidental lesion. Tiny radial scars that are completely excised within the width of a core biopsy can be classified as B2, benign.

The upgrade rate of radial scars is heavily dependent on the presence of associated atypical epithelial proliferation. Those where no epithelial atypia is seen in core biopsy have a very low rate of upgrade $(<10 \%)$, although occasional, very small series have reported a higher upgrade $\left(40 \%\right.$, two of five cases $\left.{ }^{16}\right)$. Of 410 radial scars without atypia on core biopsy in a large UK series Rakha et al.,5 reported $9 \%$ had a malignant outcome (DCIS or invasive), whilst those with atypia had an upgrade rate of $36 \%$ (comparable to the $39.5 \%$ rate for epithelial atypia in the same study). Other, older, series, notwithstanding bias in the proportion of cases that have undergone surgical excision (i.e., 102 of 198 ), show similar results (28\% upgrade for radial scar with for atypia and $4 \%$ without epithelial atypia) $;{ }^{17}$ however, some, more recent series (with similar surgical bias), show lower rates of upgrade to DCIS or invasive cancer when no atypia is seen on core biopsy ${ }^{18,19}$ although these have tended to show surprisingly high rates of associated atypical ductal hyperplasia at excision instead (e.g., $20 \%{ }^{19}$ and $\left.16 \%{ }^{18}\right)$.

Papillary lesion

Intraductal papillary lesions are composed of finger-like projections of fibrovascular cores with overlying epithelium extending into a duct lumen. They are typically classified as B3 as they may show intralesional heterogeneity. Very rarely a small papilloma may be present within the width of the core biopsy and considered completely removed by the core, and a B2, benign diagnosis can be considered. ${ }^{4}$ Conversely, particularly with the benefit of immunohistochemistry and confirmation of the absence of a myoepithelial layer between the fronds and the epithelial layer, a 
category of B4 or even B5a may be appropriate. The histopathological assessment of papillary lesions with atypia requires the pathologist to assess the size of the area of atypical epithelial proliferation present. If this is present in multiple cores, even if large samples, the area cannot be measured and distinguishing atypical epithelial proliferation within a papilloma $(<3 \mathrm{~mm}$ in extent) from low-grade DCIS within a papilloma ( $\geq 3 \mathrm{~mm}$ ) may not be possible. ${ }^{20}$

Mammograms may be normal (particularly with small intraductal papillary lesions). Other patients may present with nipple discharge or radiologically with solitary or multiple dilated ducts, a circumscribed well-defined mass (often retroareolar in location) or a cluster of calcifications (25\% cases). ${ }^{21}$ On ultrasound a well-defined solid nodule or dilated duct with intraductal mass may be identified, this latter may either fill a duct or be partially outlined by fluid. Colour Doppler ultrasound may demonstrate a vascular stalk.

As with radial scars/CSLs the most important predictor of upgrade to malignancy is the presence of associated epithelial atypia and this should be sought and recorded. When a papillary lesion is seen without any epithelial atypia, the chance of malignancy in the subsequent excision specimen is low $\left(9 \%^{5}\right.$ and $\left.13.2 \%^{22}\right)$, when atypia is present the upgrade rate is much higher $\left(36 \%{ }^{5}\right.$ and $\left.47.8 \%{ }^{22}\right)$.

\section{Cellular fibroepithelial lesion}

Although the vast majority of fibroepithelial lesions can be definitive classified as B2, i.e., benign fibroadenoma, a small proportion may have a more cellular stroma with histological features on core raising concern that the lesion may represent a phylloides tumour. Additional features include stromal overgrowth, fragmentation (defined as a stromal fragment with epithelium at one or both ends), and mitoses. 
Marked atypia of stromal cells is uncommonly seen in cores, and when present there are usually also other features suggesting phylloides tumour. Histological features on which one can definitively make a diagnosis of phylloides are uncommon but, if seen, a B3 classification is appropriate. More often the differential diagnosis lies between a cellular fibroadenoma and a benign phylloides tumour, but definite diagnosis is not possible ${ }^{4}$. Such lesions should also be designated B3 along with phrase in the histology report to indicate that "a phylloides tumour cannot be excluded".

Radiologically, fibroepithelial lesions are seen as non-specific round or oval, lobulate, generally well-circumscribed lesions with smooth margins. A radiolucent halo may be present, particularly in phylloides tumours. Calcification (typically coarse) is present in approximately $10 \%$ of lesions..$^{23}$ Ultrasonography may show septa, which are suggestive of phylloides tumour, but these are present in only a small proportion of lesions. ${ }^{24}$

A lesion that is definitively diagnosed as a phylloides tumour warrants different clinical management to one where differential lies between a cellular fibroadenoma and a phylloides tumour, although both are classified as B3 lesions. The former merits surgical excision with a margin (locally designated) of normal tissue whilst the latter may be enucleated; however, it should be noted that only a very small proportion of lesions diagnosed as cellular fibroepithelial lesion are malignant in the excision specimen. ${ }^{25} \mathrm{~A}$ more useful measure of the "upgrade rate" is the proportion that are a phylloides tumour on excision; the figures in the literature show wide variation even in the larger series between $16 \%{ }^{2}$ and $76 \%{ }^{25}$ This implies some variation in the application of diagnostic criteria. One typical study found that $37 \%$ of cellular fibroepithelial lesions on core were phylloides tumours on excision, but significantly only one of the 52 lesions was a malignant phylloides tumour. ${ }^{26}$ 


\section{Mucocoele-like lesion}

Mucin extrusion into the stroma (a mucocoele-like lesion) can be associated with benign cysts, $\mathrm{ADH}, \mathrm{DCIS}$, or invasive carcinoma, particularly of mucinous type. As in papillary lesions and radial scars/CSLs, the upgrade rate is related to the presence of associated epithelial atypia, albeit in small series. Rakha et al., ${ }^{27}$ reported that combining their series with the literature, six of 162 patients (4\%) with mucocoele-like lesion without atypia on core biopsy had malignancy in the subsequent excision specimen, whilst if atypia was seen, the upgrade rate was $21 \%$ (seven of 33 patients).

\section{Other indeterminate B3 lesions}

There are some other rare lesions that are best classified as B3 on core biopsy such as adenomyoepithelioma, microglandular adenosis, spindle cell lesions either of uncertain diagnosis on core or spindle cell lesions with definitive diagnosis, such as fibromatosis, myofibroblastoma, nerve sheath tumours, nodular fasciitis, and vascular lesions that are difficult to classify. Other spindle cell lesions, such as pseudoangiomatous stromal hyperplasia (PASH) can be definitively diagnosed as benign, B2, and are not considered here. The upgrade rate of a range of these uncommon indeterminate B3 lesions remains unclear as there are insufficient data in the literature from which to draw conclusions. These, typically mass-forming lesions, are difficult to assess radiologically and histologically, and at present, it is regarded prudent to remove these surgically; however, each lesion must be considered in context; some spindled cell lesions potentially require complete excision with a rim of surrounding tissue, whereas others benefit from surgical excision for diagnostic purposes. 


\section{B4 diagnoses}

Although these guidelines are targeted at the management of B3 lesions, mention is merited of B4, suspicious diagnoses. The commonest reason for a B4 diagnosis is for lesions suspicious of DCIS. ${ }^{26}$ A B4 category is given to cores where there are technical problems, such as crushed or poorly fixed tissues that contain probable carcinoma, but insufficient features for definitive diagnoses. Other situations include those where small groups of apparently neoplastic cells are seen within a blood clot or adherent to the outer aspect of the sample. Very small foci suspicious of invasive carcinoma in which there is insufficient material to allow full assessment are also regarded as B4, suspicious. In general, repeat of the sampling procedure (14 G or vacuum-assisted sampling) is likely to provide sufficient material in such circumstances to allow the histopathologist to reach a definitive malignant diagnosis and these rare cases do not usually provide the same conundrum of management as the B3 lesions.

\section{RECOMMENDATIONS}

Given the differing upgrade rates to malignancy for various B3 lesion presented above (i.e., ranging from $<2 \%$ for fibroepithelial lesion to $39.5 \%$ for epithelial atypia/AIDEP in a large series of 1,548 B3 core biopsies ${ }^{5}$ ) it is evident that a case-bycase approach is required. We, nevertheless, consider it inappropriate to leave the vast majority of $\mathrm{B} 3$ lesions in the breast when diagnosed with $14 \mathrm{G}$ or VAB, without further histological evaluation. It is also clear that the upgrade rates of some lesions that are classified as B3 are low. Diagnostic surgical excision (often under wirelocalised guidance) has historically been performed for the vast majority of lesions 
considered suspicious or malignant radiologically when definitive diagnosis has proven impossible with cytology or histology. Standards for open biopsy include guidance that the fresh weight of tissue removed for all cases in which a diagnostic open surgical biopsy is performed should be recorded and that $\geq 90 \%$ of open surgical biopsies carried out for diagnosis, which prove to be benign should weigh $\leq 20 \mathrm{~g} .{ }^{28}$

The specific management recommendations from this group for the different forms of B3 lesion are shown in Figs. 1-6. We consider vacuum-assisted excision (VAE)/thorough sampling the overall method of choice for the detailed secondary assessment of most B3 lesions, whether initially diagnosed on core biopsy or primary diagnostic VAB. There are, however, some specific exceptions. Papillary lesions with atypia identified in the core biopsy/VAB specimen require assessment of the extent in continuity of the atypia ${ }^{20}$ and thus examination of the intact specimen is preferred. Similarly, lesions that are difficult to diagnose histologically are best excised as one portion of tissue. Clearly, all cases should be discussed at a multidisciplinary team meeting (MDTM) and dealt with on an individual basis.

Vacuum-assisted techniques are an alternative and cost-effective non-surgical method for the provision of a greater volume of tissue for histological evaluation and have been applied diagnostically in some series, although often there is an admixture of different gauge core biopsies in series making data unclear. VAB (under ultrasound of stereotactic guidance), with appropriate training, ${ }^{29}$ is suitable for sampling a wide variety of lesions and has high patient acceptance. ${ }^{30}$ The aim of the procedure may be to provide more tissue (VAB) than with a 14 or $16 \mathrm{G}$ core, or it may be to remove the entire lesion (VAE). VAE of benign lesions has been approved by the National Institute for Health and Care Excellence (NICE) (https://www.nice.org.uk/guidance/ipg156/chapter/1-guidance). 
As a diagnostic procedure, the purpose of $\mathrm{VAB}$ is to obtain representative sampling of a lesion; if a lesion is small $(5-10 \mathrm{~mm})$ it may be removed in its entirety but this is not the aim. The purpose of VAE is ideally to remove the lesion in its entirety (e.g., if $\leq 15 \mathrm{~mm}$ ); however, areas of mammographic change that include B3 diagnoses range from a few millimetres, to several centimetres. Planning VAE or surgical excision of areas $<20 \mathrm{~mm}$ is straightforward, but it is more difficult to ensure that sampling is sufficiently thorough in larger areas of mammographic change. It should be remembered that diagnostic surgery would not be expected to remove a large lesion entirely, and therefore, the aim of a vacuum-assisted procedure is, similarly, to sample sufficiently to ensure that the lesion has been reasonably represented and the presence of associated malignancy excluded.

It is difficult to define what represents "thorough" sampling of a large lesion that cannot be completely excised, and clearly, this will be influenced by the size and nature of the radiological abnormality. If the lesion cannot be excised by VAE the authors consider it appropriate to remove a sample of comparable volume/weight to a diagnostic surgical excision and recommend that at least $12 \times 7 \mathrm{G}$ cores are removed, representing approximately $4 \mathrm{~g}$, depending on the nature (fatty or fibroglandular) of the tissue excised. The weight of the VAE cores can either be recorded or can be estimated by the total number of cores taken and estimated weight per core from needle gauge used. As a minimum, the number and gauge of the cores should be recorded. Weights and numbers of cores equivalent to $4 \mathrm{~g}$ of turkey breast tissue are shown in Table 1.

For extensive lesions (e.g., widespread microcalcifications), VAE from more than one area should be considered, targeted appropriately according to the radiological 
features. Further sampling should be directed towards areas of greater radiological concern, preferably distant from the site of the first specimen. The area to target for some radiological lesions may be the periphery (e.g., radial scar) but for others there is no evidence that any co-existing unsuspected malignancy is likely to be in a specific zone of the radiological abnormality. Of note, larger CSLs may be technically difficult to excise with vacuum-assisted technique and sampling of more than one area is considered the most pragmatic approach. On the whole, the authors consider sampling to be adequate if at least one-third of an extensive area of change is removed. This is most commonly an area of low suspicion microcalcification and may represent a columnar cell process with flat epithelial atypia as a component; it is reassuring that, even if present, any malignancy associated with such areas is typically low-grade (DCIS or invasive) disease. ${ }^{31}$

Although the approach recommended here for management of B3 lesions by VAE rather than surgical excision has not been widely utilised, there is evidence from some units that this does indeed reduce the number of benign open surgical biopsies $^{32,33}$ and is safe. In one of the few series presenting data from this approach, of 398 patients with B3 lesions who were suitable, 321 had "second-line" VAB, 24\% subsequently required surgery and 245 avoided surgery; more significantly, at 3 years follow-up no patients had cancer at the B3 biopsy site. ${ }^{34}$ It seems obvious that findings at VAE should be reported in conjunction with the initial diagnostic biopsy (core or $\mathrm{VAB}$ ) as a summation of the samples from a particular radiological lesion. The report should comment on whether similar changes are present in both diagnostic and excision specimens, and whether there are signs of previous biopsy in the excision indicating that the appropriate site has been sampled. As the differential diagnosis between AEDIP and low-grade DCIS includes the 
number of duct spaces involved by a low-grade cytologically and architecturally atypical process, all specimens should be evaluated and an overall diagnosis from several procedures may be required.

Some additional practical issues merit comment. Firstly, distinguishing VAB from VAE has implications, not only for analysis of the data in the literature, but also for communication between radiologists and pathologists and also for coding within data collection systems; VAB, similar to $14 \mathrm{G}$ core biopsy, is a biopsy procedure, part of the triple approach, and requires a "B" code (in this case B3), the aim of VAE is to excise or thoroughly sample the lesion and is regarded as akin to a surgical diagnostic excision and does not require a biopsy ("B") code. Thus, the radiologist must be clear, both in their intention when performing the procedure and in communication with the pathologist and data entry/administrative colleagues. Secondly, after either VAB or VAE, marker clip(s) should be deployed and a mammogram performed to check the positioning and this should be documented in the report.

Importantly, if core biopsy diagnosis and radiological features are not concordant then the recommended course of action will be different from when these are in agreement. For example, if the radiological lesion is a well-defined mass whereas the core biopsy shows an AIDEP or lobular neoplasia, a repeat core, rather than necessarily turning automatically to second-line diagnostic $\mathrm{VAB}$, may be considered appropriate; an atypical intraductal epithelial proliferation is unlikely to produce a welldefined mass lesion and this discordance implies that the index lesion was missed on core. Conversely, if there remains non-concordance of radiological and pathological assessment after needle core biopsy and subsequent $\mathrm{VAB}$, then diagnostic surgery 
should be considered for definitive diagnosis, unless the lesion has unequivocally been completely excised by VAE.

The national breast screening pathology audit 2015 (unpublished) has shown variation in the frequency of B3 diagnoses (3.3-12.6\%) between NHSBSP units. Although this is undoubtedly, at least partly, related to variation in the application of histological features and categories, there are also differences in recall and biopsy rates by radiology teams. It is essential that there is ongoing audit of B3 diagnosis and management in order that these recommendations are applied safely and that this approach for reducing unnecessary benign biopsies in women attending for national breast screening is both safe and effective. In particular, this writing group considers it essential that this is "joined-up" between the NHS BSP and surgical services to obtain robust data.

Although, as noted above, it is apparent that the B3 lesions have a range of risk of upgrade to DCIS or invasive carcinoma, those with associated epithelial atypia also confer an increase in risk of development of carcinoma in either breast over the subsequent years. For ALH and LCIS and for true ADH, the risk is well recognised. For FEA, the degree of risk is less clear, but is almost certainly low, and requires further research. Nevertheless, following adequate investigation of the radiological abnormality at the time of presentation, the group considers mammographic surveillance to be appropriate for women who have presented with a B3 lesion with epithelial atypia who are at moderately increased risk of subsequent breast carcinoma. In the first instance, this same approach is recommended for FEA, but this merits review in due course. There remains a lack of clarity, however, regarding the optimal interval (annual or 18-monthly) and the length of time for which mammographic surveillance should continue and, in particular, whether this should be 
tailored according to the nature of the risk lesion (LN or ADH or FEA), patient age at diagnosis, breast density, associated family history, or other factors. It is not within the remit of these authors to advise on this complex issue or to recommend where such surveillance should be undertaken. Pragmatically therefore, the schematic diagrams presented, suggest annual mammography, as this is undertaken at present by most units in the UK. This protocol for surveillance should, we believe, be kept under review and amended as more data and national guidance become available.

In summary, the aim of this document is to present a review and guidance on the management of B3 lesions of the breast. These guidelines reflect suggested practice as stated by the NHS BSP and approved by the RCR.

It is essential that a multidisciplinary approach is applied and that there is close communication within the team, particularly between pathologists and the radiologists, regarding these potentially problematic lesions; however, the upgrade rates from review of the literature for each of the B3 lesions indicate that these warrant further examination, whether seemingly co-incidental or interpreted as the cause of radiological abnormality. It is the view of this group that for the majority of these lesions, thorough sampling by $\mathrm{VAB}$ technique (akin to excision) can safely be used following initial diagnostic sampling (by either $14 \mathrm{G}$ or VAB), and allows the patient to avoid a surgical procedure and is cost-effective. Clearly, some of these lesions are those that are also associated with a longer-term increased risk of development of carcinoma in either breast (as well as concurrent upgrade) and the recommendations regarding follow-up surveillance for such lesions should follow updated national guidance.

\section{REFERENCES}


1. Bianchi S, Caini S, Renne G, et al. VANCB Study Group. Positive predictive value for malignancy on surgical excision of breast lesions of uncertain malignant potential (B3) diagnosed by stereotactic vacuum-assisted needle core biopsy (VANCB): a large multi-institutional study in Italy. Breast. 2011 Jun;20(3):264-70.

2. El-Sayed ME, Rakha EA, Reed J, Lee AH, Evans AJ, Ellis IO. Predictive value of needle core biopsy diagnoses of lesions of uncertain malignant potential (B3) in abnormalities detected by mammographic screening. Histopathology. 2008 Dec;53(6):650-7.

3. Maclean GM, Courtney SP, Umeh H, Sanjeev S, McCormick C, Smith BM. Is mode of presentation of B3 breast core biopsies (screen-detected or symptomatic) a distinguishing factor in the final histopathologic result or risk of diagnosis of malignancy? World J Surg. 2013 Nov;37(11):2607-12.

4. Lee AHS, Anderson N, Carder P, et al. Guidelines for non-operative diagnostic procedures and reporting in breast cancer screening. Royal College of Pathologists. June 2016. Available at: https://www.rcpath.org/resourceLibrary/g150-non-opreporting-breast-cancer-screening-jun16-pdf.html. Accessed ????

Commented [SD2]: Provide date

5. Rakha EA, Lee AH, Jenkins JA, Murphy AE, Hamilton LJ, Ellis IO. Characterization and outcome of breast needle core biopsy diagnoses of lesions of uncertain malignant potential (B3) in abnormalities detected by mammographic screening. Int $\mathrm{J}$ Cancer. 2011 Sep 15;129(6):1417-24. 
6. Yu YH, Liang C, Yuan XZ. Diagnostic value of vacuum-assisted breast biopsy for breast carcinoma: a meta-analysis and systematic review. Breast Cancer Res Treat. 2010 Apr;120(2):469-79.

7. Canas-Marques R, Schnitt SJ. E-cadherin immunohistochemistry in breast pathology: uses and pitfalls. Histopathology. 2016 Jan;68(1):57-69. doi: 10.1111/his.12869.

8. Carder PJ, Shaaban A, Alizadeh Y, Kumarasuwamy V, Liston JC, Sharma N. Screen-detected pleomorphic lobular carcinoma in situ (PLCIS): risk of concurrent invasive malignancy following a core biopsy diagnosis. Histopathology. 2010 Sep;57(3):472-8. doi: 10.1111/j.1365-2559.2010.03634.x.

9. Menon S, Porter GJ, Evans AJ, Ellis IO, Elston CW, Hodi Z, Lee AH. The significance of lobular neoplasia on needle core biopsy of the breast. Virchows Arch. 2008 May;452(5):473-9. doi: 10.1007/s00428-008-0607-8.

10. Buckley ES, Webster F, Hiller JE, Roder DM, Farshid G A systematic review of surgical biopsy for LCIS found at core needle biopsy — do we have the answer yet? Eur J Surg Oncol. 2014 Feb;40(2):168-75.

11. Hussain M, Cunnick GH. Management of lobular carcinoma in-situ and atypical lobular hyperplasia of the breast-a review. Eur J Surg Oncol. 2011 Apr;37(4):279-89.

12. Hwang H, Barke LD, Mendelson EB, Susnik B. Atypical lobular hyperplasia and 
classic lobular carcinoma in situ in core biopsy specimens: routine excision is not necessary. Mod Pathol. 2008 Oct;21(10):1208-16.

13. Murray MP, Luedtke C, Liberman L, Nehhozina T, Akram M, Brogi E. Classic lobular carcinoma in situ and atypical lobular hyperplasia at percutaneous breast core biopsy: outcomes of prospective excision. Cancer. 2013;119:1073-9.

14. Fraser JL, Raza S, Chorny K, Connolly JL, Schnitt SJ. Columnar alteration with prominent apical snouts and secretions: a spectrum of changes frequently present in breast biopsies performed for microcalcifications. Am J Surg Pathol. 1998 Dec;22(12):1521-7.

15. Verschuur-Maes $\mathrm{AH}$, van Deurzen $\mathrm{CH}$, Monninkhof EM, van Diest PJ. Columnar cell lesions on breast needle biopsies: is surgical excision necessary? A systematic review. Ann Surg. 2012 Feb;255(2):259-65.

16. Jackman RJ, Nowels KW, Rodriguez-Soto J, Marzoni FA Jr, Finkelstein SI, Shepard MJ. Stereotactic, automated, large-core needle biopsy of nonpalpable breast lesions: false-negative and histologic underestimation rates after long-term follow-up. Radiology. 1999 Mar;210(3):799-805.

17. Brenner RJ, Jackman RJ, Parker SH, et al. Percutaneous core needle biopsy of radial scars of the breast: when is excision necessary? AJR Am J Roentgenol. 2002 Nov;179(5):1179-84. 
18. Donaldson AR, Sieck L, Booth CN, Calhoun BC. Radial scars diagnosed on breast core biopsy: frequency of atypia and carcinoma on excision and implications for management. Breast. 2016 Jun 29. pii: S0960-9776(16)30081-9. doi: 10.1016/j.breast.2016.06.007.

19. Li Z, Ranade A, Zhao C. Pathologic findings of follow-up surgical excision for radial scar on breast core needle biopsy. Hum Pathol. 2016 Feb;48:76-80. doi: 10.1016/j.humpath.2015.06.028.

20. Page DL, Salhany KE, Jensen RA, Dupont WD. Subsequent breast carcinoma risk after biopsy with atypia in a breast papilloma. Cancer. 1996;78:258-66

21. Eiada R, Chong J, Kulkarni S, Goldberg F, Muradali D. Papillary lesions of the breast: MRI, ultrasound, and mammographic appearances. AJR Am J Roentgenol. 2012 Feb;198(2):264-71.

22. Bianchi S, Bendinelli B, Saladino V, et al. Non-malignant breast papillary lesions-B3 diagnosed on ultrasound-guided 14-gauge needle core biopsy: analysis of 114 cases from a single institution and review of the literature. Pathol Oncol Res. 2015 Jul;21(3):535-46. doi: 10.1007/s12253-014-9882-7.

23. Wiratkapun C, Piyapan P, Lertsithichai $P$, Larbcharoensub N. Fibroadenoma versus phylloides tumor: distinguishing factors in patients diagnosed with fibroepithelial lesions after a core needle biopsy. Diagn Interv Radiol 2014; 20:27-33 
24. Evans AJ, Wilson ARM, Blamey RW, Robertson JFR, Ellis IO, Elston CW. Phylloides tumour. In Atlas of breast disease management: 50 illustrative cases. London: W B Saunders, 1998; 69-70

25. Abdulcadir D, Nori J, Meattini I, et al. Phylloides tumours of the breast diagnosed as B3 category on image-guided 14-gauge core biopsy: analysis of 51 cases from a single institution and review of the literature. Eur J Surg Oncol. 2014 Jul;40(7):859-64. doi: 10.1016/j.ejso.2014.02.222.

26. Rakha EA, Ho BC, Naik V, et al. Outcome of breast lesions diagnosed as lesion of uncertain malignant potential (B3) or suspicious of malignancy (B4) on needle core biopsy, including detailed review of epithelial atypia. Histopathology. 2011 Mar;58(4):626-32.

27. Rakha EA, Shaaban AM, Haider SA, et al. Outcome of pure mucocele-like lesions diagnosed on breast core biopsy. Histopathology. 2013;62:894-898

28. Association of Breast Surgery at BASO. Quality assurance guidelines for surgeons in breast cancer screening (M Sibbering, R Watkins, J Winstanley, J Patnick, eds). NHSBSP Publication no. 20, $4^{\text {th }}$ edition. March 2009 
29. The Royal College of Radiologists. 2012. Ultrasound training recommendations for medical and surgical specialties. $2^{\text {nd }}$ edition. Board of the Faculty of Clinical Radiology.

Available

at:

https://www.rcr.ac.uk/sites/default/files/publication/BFCR(12)17 ultrasound training.p

df. Accessed ?????

Commented [SD3]: Provide date

30. Huber S, Wagner M, Medl M, Czembirek H. Benign breast lesions: minimally invasive vacuum-assisted biopsy with 11-gauge needles-patient acceptance and effect on follow-up imaging findings. Radiology 2003; 226:783-790

31. Abdel-Fatah TM, Powe DG, Hodi Z et al. High frequency of coexistence of columnar cell lesions, lobular neoplasia, and low grade ductal carcinoma in situ with invasive tubular carcinoma and invasive lobular carcinoma. Am J Surg Pathol. 2007 Mar;31(3):417-26.

32. Rajan S, Shaaban AM, Dall BJ, Sharma N. New patient pathway using vacuumassisted biopsy reduces diagnostic surgery for B3 lesions. Clin Radiol. 2012 Mar;67(3):244-9. doi: 10.1016/j.crad.2011.09.002.

33. Pieri A, Hemming D, Westgarth J, Lunt L. Vacuum-assisted biopsy is a viable alternative to surgical biopsy in the investigation of breast lesions of uncertain malignant potential. Surgeon. 2015 Oct 31. pii: S1479-666X(15)00100-6. doi: 10.1016/j.surge.2015.10.001. 
34. Strachan C, Horgan K, Millican-Slater RA, Shaaban AM, Sharma N. Outcome of a new patient pathway for managing B3 breast lesions by vacuum-assisted biopsy: time to change current UK practice? J Clin Pathol. 2016 Mar;69(3):248-54. doi: 10.1136/jclinpath-2015-203018.

35. Preibsch H, Baur A, Wietek BM, et al. Vacuum-assisted breast biopsy with 7gauge, 8-gauge, 9-gauge, 10-gauge, and 11-gauge needles: how many specimens are necessary? Acta Radiol. 2015;56(9):1078-84.

Figure 1. Suggested pathway for management of patients with AIDEP and for radial scar/CSL with atypia. *The MDTM should, in particular, consider how representative the sampling is and how worrying the pathology is (taking into account the summation of the 14 G/VAB and VAE specimens) in decision-making. It is anticipated that most patients will undergo diagnostic surgical excision in this situation, but if suspicion is low consideration may be given to annual mammographic surveillance. ** In the context of a low cytonuclear grade proliferation such as this, the LORIS clinical trial could also be considered.

Figure 2. Suggested pathway for management of patients with LN. *The MDTMs should, in particular, consider whether there is radiological-pathological concordance (14 G and/or vacuum-assisted sampling at all stages) but if there is radiological suspicion of malignancy and initial biopsy shows minor LN only, repeat sampling can be considered rather than VAE. 
Figure 3. Suggested pathway for management of patients with FEA. *The data indicating subsequent risk of development of invasive carcinoma after surgical excision showing FEA alone suggests a low probability of progression. At present many units undertake annual surveillance mammography of these women and in the setting of VAE/thorough sampling, the writing group considered this approach prudent. ${ }^{\star \star}$ The MDTM should, in particular, consider the extent, degree, and suspicion of the atypia seen in the VAE. If unequivocal architectural atypia is present in the VAE (i.e., akin to AIDEP) patients may undergo diagnostic surgical excision; however, if suspicion is low consideration may be given to annual mammographic surveillance.

Figure 4. Suggested pathway for management of patients with radial scar or papillary without atypia in initial biopsy. *A similar approach for mucocoele-like lesion without atypia is also suggested; if atypia is seen in association with mucocoele-like lesion management as per AIDEP is recommended. ${ }^{* *}$ The MDTM should, in particular, consider the nature, degree, and suspicion of the atypia seen in the VAE.

Figure 5. Suggested pathway for management of patients with papillary lesion with atypia in initial biopsy. ${ }^{*}$ This pathway differs from others as surgical diagnostic excision is recommended following initial $14 \mathrm{G}$ core or VAB, because of the need for the pathologist to assess the continuity and measurement of low-grade atypia in a papillary lesion to distinguish low-grade DCIS in a papilloma ( $\geq 3 \mathrm{~mm}$ ) from atypia $(<3 \mathrm{~mm}) \cdot{ }^{20}{ }^{\star *}$ The MDTM should, in particular, consider the nature of the atypia seen and whether this is only present within the papillary lesion or also in the adjacent tissue. 
Figure 6. Suggested pathway for management for other indeterminate B3 lesions.

The surgical approach will vary according to histological features, e.g., enucleation for cellular fibroepithelial lesion compared to excision with rim of normal tissue for definite phylloides tumour.

Table 1. Mean weight cores from turkey breast phantom, standard deviation, and numbers equivalent to $4 \mathrm{~g}$ tissue. ${ }^{35}$

\begin{tabular}{|c|c|c|c|}
\hline $\begin{array}{l}\text { Core and } \\
\text { manufacturer }\end{array}$ & $\begin{array}{l}\text { Weight of } \\
\text { one core }(g)\end{array}$ & $\begin{array}{l}\text { Standard deviation } \\
\text { of weight on one } \\
\text { core }(\mathrm{g})\end{array}$ & $\begin{array}{l}\text { No. of cores } \\
\text { equating to } \\
\text { approximately } 4 \mathrm{~g}\end{array}$ \\
\hline $\begin{array}{l}\text { 11-G Original } \\
\text { Mammotome }\end{array}$ & 0.084 & 0.032 & 48 \\
\hline 10-G Vacora & 0.142 & 0.006 & 28 \\
\hline $\begin{array}{l}\text { 10-G EnCore } \\
\text { Enspire }\end{array}$ & 0.221 & 0.039 & 18 \\
\hline $\begin{array}{l}\text { 9-G ATEC Sapphire } \\
\text { needle }\end{array}$ & 0.121 & 0.014 & 33 \\
\hline $\begin{array}{l}\text { 8-G Original } \\
\text { Mammotome }\end{array}$ & 0.192 & 0.027 & 21 \\
\hline $\begin{array}{l}\text { 8-G Mammotome } \\
\text { Revolve }\end{array}$ & 0.334 & 0.046 & 12 \\
\hline $\begin{array}{l}\text { 7-G EnCore } \\
\text { Enspire }\end{array}$ & 0.363 & 0.053 & 11 \\
\hline
\end{tabular}


Atypical Intraductal Epithelial Proliferation (AIDEP) or radial scar/CSL with atypia

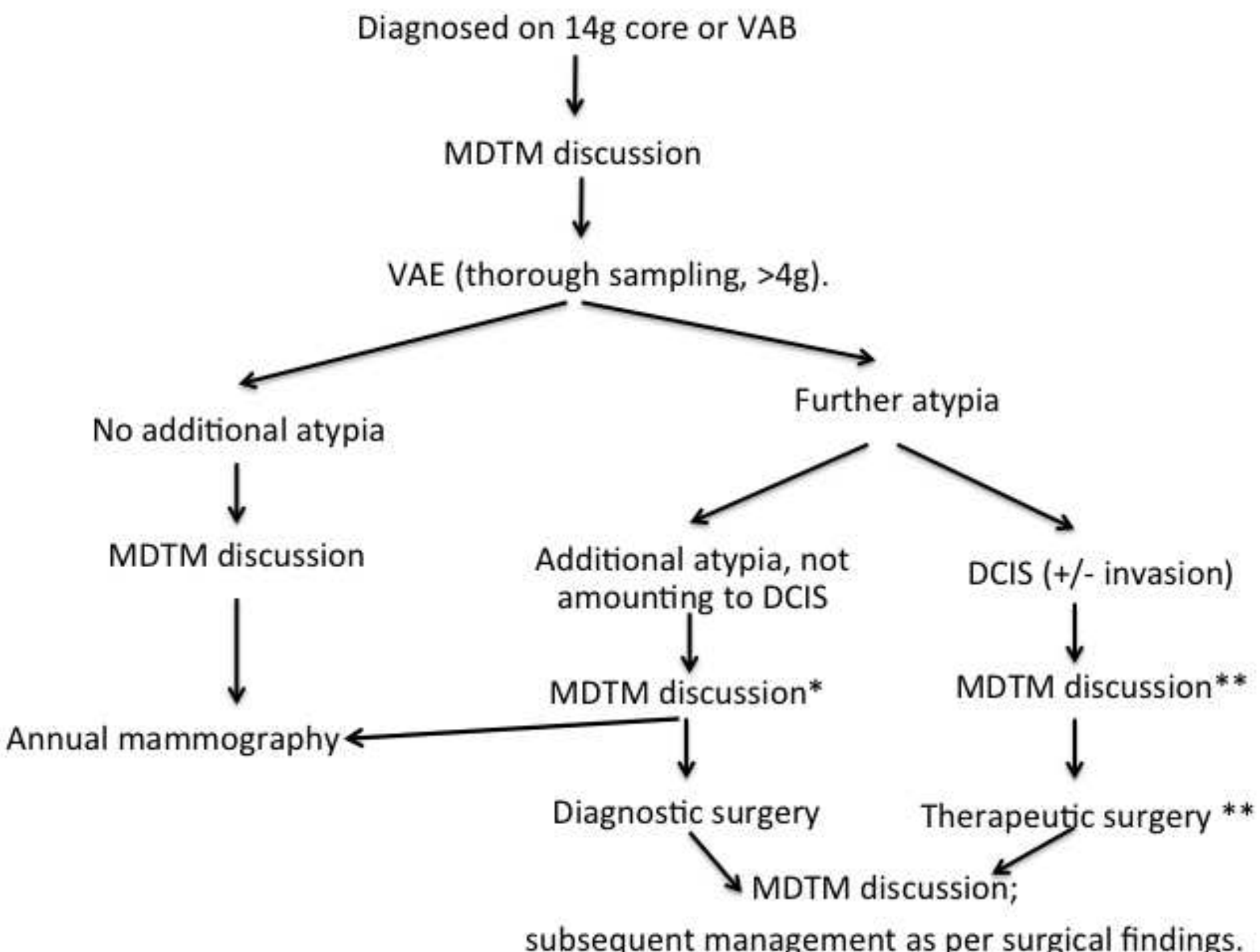

Diagnosed on $14 \mathrm{~g}$ core or VAB

MDTM discussion

VAE (thorough sampling, $>4 \mathrm{~g}$ ).

No additional atypia

MDTM discussion

subsequent management as per surgical findings. 


\title{
Classical lobular neoplasia (LN)
}

no comedo-type necrosis, not mass-forming, not pleomorphic type

Diagnosed on $14 \mathrm{~g}$ core or VAB
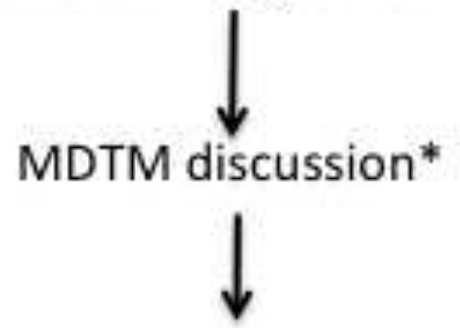

VAE (thorough sampling, $>4 \mathrm{~g}$ )

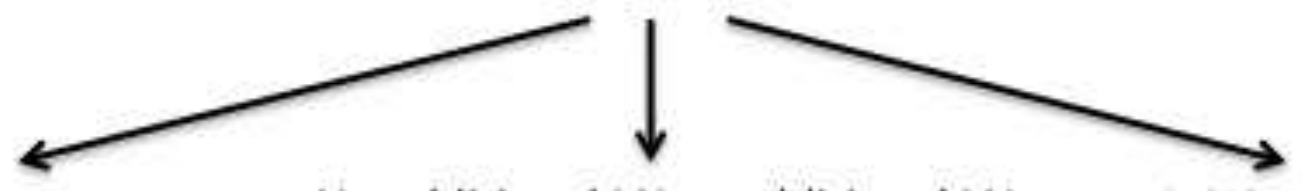

No additional LN or additional LN

No additional $\mathrm{LN}$ or additional $\mathrm{LN}$ only. Radiological-pathological concordance. only. Radiology not suspicious of malignancy.

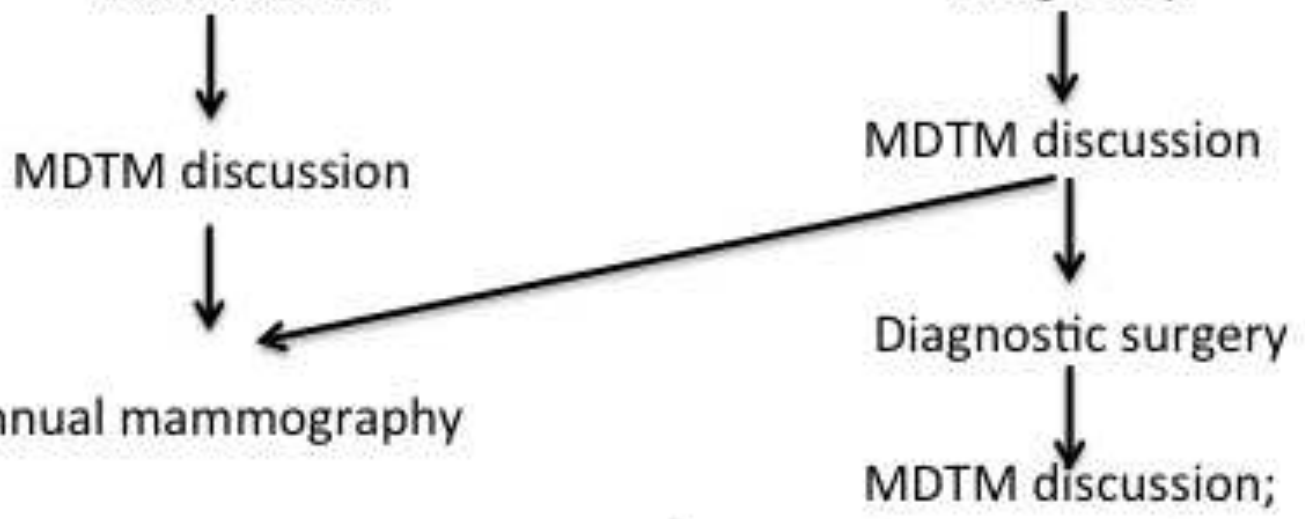

subsequent management as per surgical findings.

Annual mammography if no DCIS or $\mathrm{DCIS}+$ /- invasive carcinoma, or pleomorphic LCIS

Annual mammography

\author{
invasive carcinoma.
}




\section{Flat epithelial atypia (FEA)}

Diagnosed on $14 \mathrm{~g}$ core or $\mathrm{VAB}$

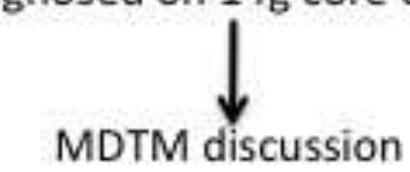

VAE (thorough sampling, $>4 \mathrm{~g}$ ).

If large area of microcalcification take samples from more than one area.

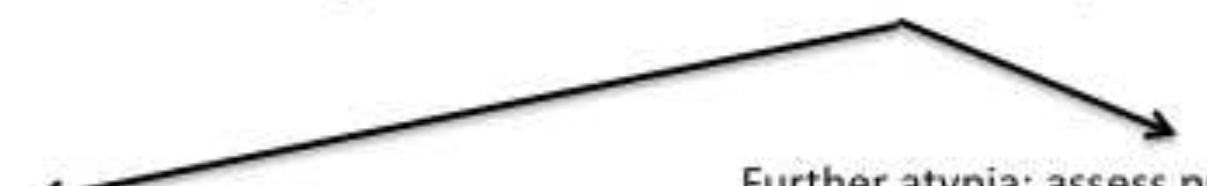

Further atypia; assess presence of architectural atypia

No additional atypia
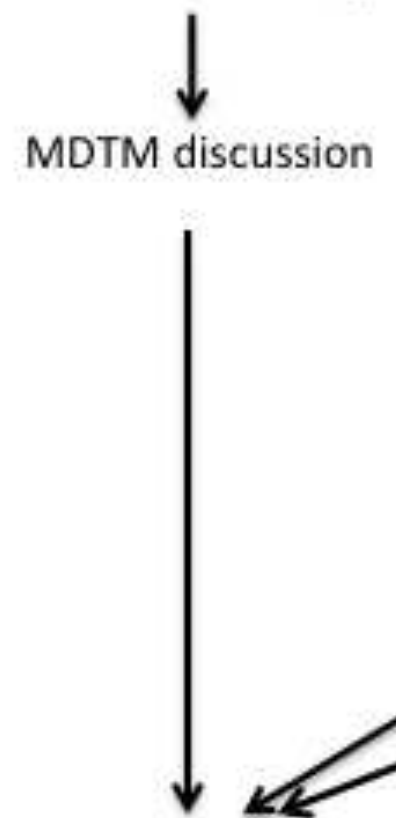

Annual mammography*

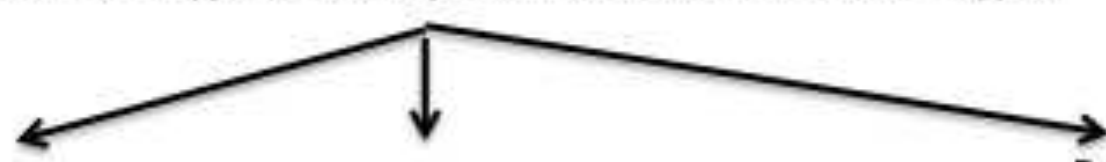

Additional, including

Additional FEA, nil worse

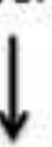

MDTM discussion

MDTM discussion**

architectural, atypia (see AIDEP)

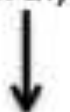

Diagnostic surgery

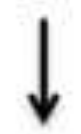

MDTM discussion;

subsequent management as per surgical findings. Annual mammography if no DCIS or invasive carcinoma
DCIS +/-invasive cancer<smiles>[14CH3]</smiles>

MDTM discussion<smiles>C[AlH]</smiles>

Therapeutic surgery<smiles>C[12CH3]</smiles>

MDTM discussion;

subsequent management as per surgical findings. 
B3, radial scar or papillary lesion or mucocoele-like lesion*, without atypia
Diagnosed on $14 \mathrm{~g}$ core or VAB

B3, radial scar or papillary lesion or mucocoele-like $\mathrm{I}$
Diagnosed on $14 \mathrm{~g}$ core or $\mathrm{VAB}$

Papillary lesion with atypia

Diagnosed on $14 \mathrm{~g}$ core or VAB

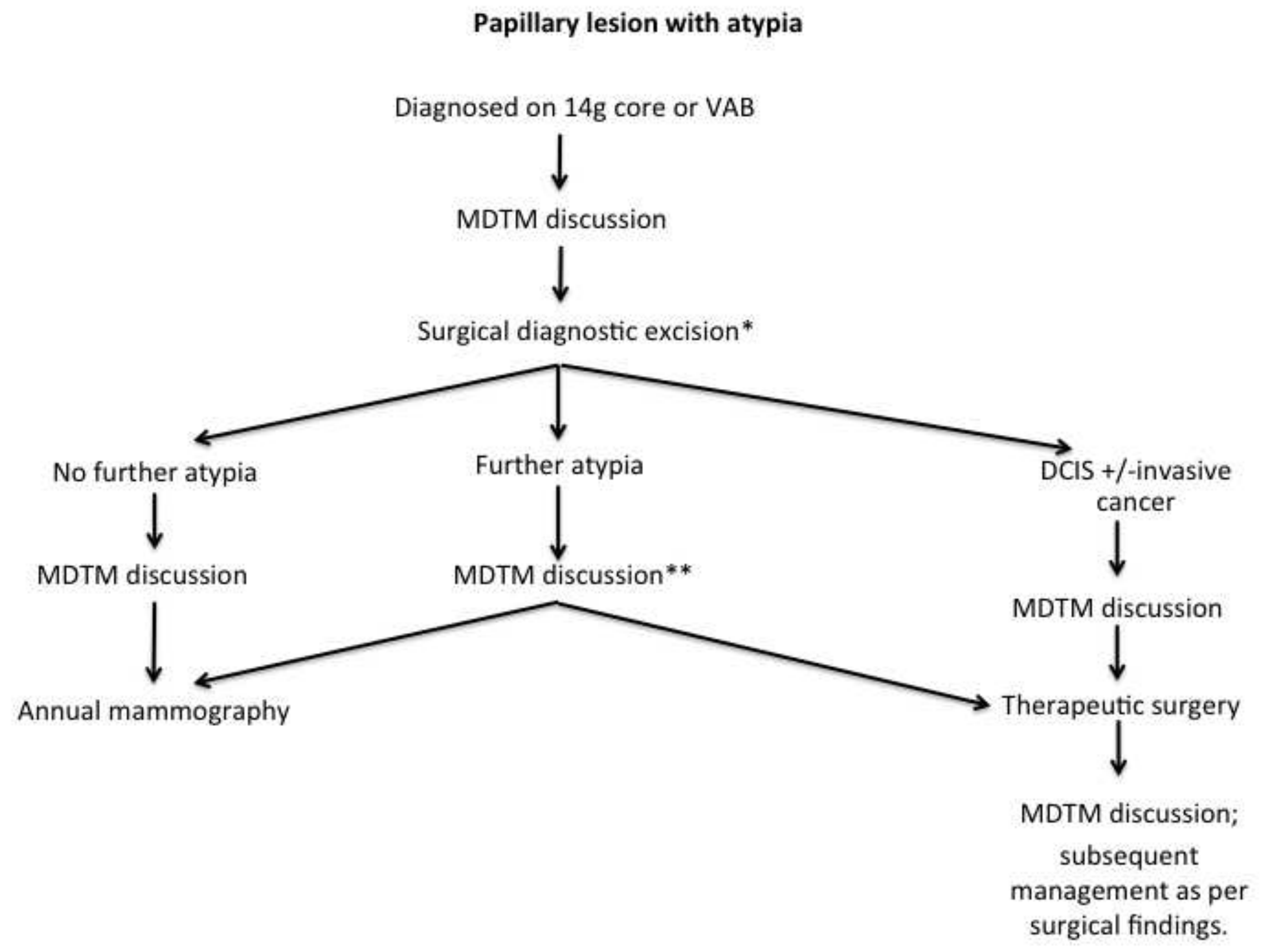


Highlights

NOT REQUIRED

Highlights 